\title{
New Initial Parameter for the Constrained Optimization Method
}

\author{
Abbas Y. Al-Bayati \\ Ban Ahmed Mitras \\ profabbasalbayati@yahoo.com \\ dr.banah.mitras@gmail.com \\ College of Computer Sciences and Mathematics \\ University of Mosul, Iraq
}

Received on: 16/02/2005

Accepted on: 30/05/2005

\section{ABSTRACT}

In this paper, we have investigated a new initial parameter in the nonlinear constrained optimization method. The aim of this new method is to make a balance between interior and exterior method for constrained optimization. The new technique has been programmed to solve some of standard problems in the non-linear optimization. The results are too effective when compared with other standard optimization methods like interior and exterior methods.

Keyword: constrained optimization, penalty method, Barrier method.

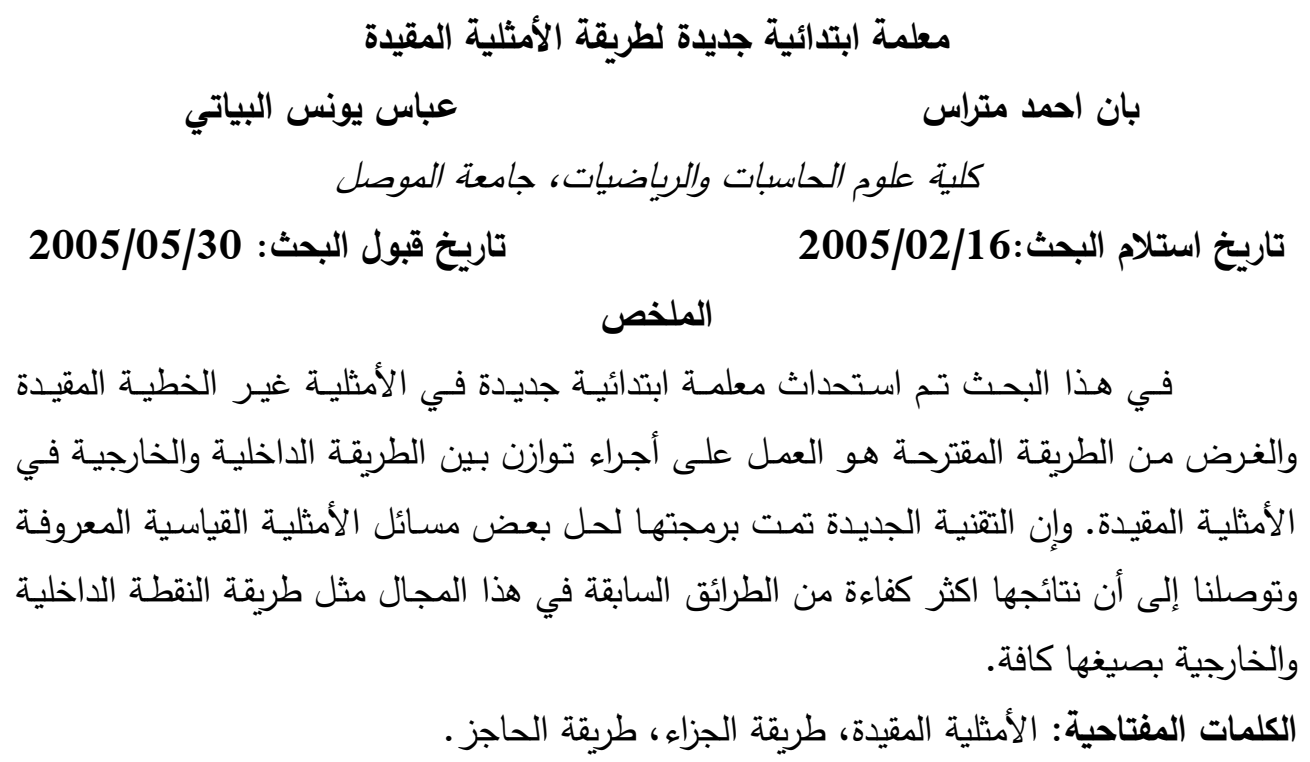

\section{Introduction:}

Consider the constrained optimization problem

Minimize $f(x)$

Where $\mathrm{x}$ required satisfying the general equality constraints

$$
c_{i}(x)=0 \quad 1 \leq i \leq m
$$

and the inequality constraints

$$
c_{i}(x) \geq 0 \quad m+1 \leq i \leq n
$$


where $f$ and $c_{i}$ map $R^{n}$ into $R$. We assume that $f(x)$ and the $c_{i}(x)$ are twice continuously differentiable. (Toint et. al., 1997)

In this paper we have used exterior-interior point method. The exterior method is used for equality and inequality constraints with the new objective function:

$$
\phi_{k}(x, r)=f(x)+g\left(r_{k}\right) \alpha\left(x_{k}\right)
$$

where $g\left(r_{k}\right)$ is a function of parameter $\alpha\left(x_{k}\right)$ and the remainder of the second term is the penalty function.

The interior method is suitable for equality constraints with the new objective function:

$$
\phi_{k}(x, r)=f(x)+g\left(r_{k}\right) B\left(x_{k}\right)
$$

where $g\left(r_{k}\right)$ is a function of parameter $B\left(x_{k}\right)$, the second term is the Barrier function. (Abdy and Dempster, 1983)

Although both exterior and interior-point methods have many points of similarity, they represent two different points of view. In an exteriorpoint procedure, we start from an infeasible point and gradually approach feasibility. While doing so, we move away from the unconstrained optimum of the objective function. In an interior-point procedure, we start at a feasible point and gradually improve our objective function, while maintaining feasibility. The requirement that we begin at a feasible point and remain within the interior of the feasible inequality constrained region is the chief difficulty with interior-point methods. In many problems we have no easy way to determine a feasible starting point, and a separate initial computation may be needed. Also, if equality constraints are present, we do not have a feasible inequality constrained region in which to maneuver freely. Thus interior-point methods cannot handle equalities. (Biggs, 1989)

We many readily handle equalities by using a "mixed" method in which we use interior-point penalty functions for inequality constraints only. Thus, if the first $m$ constraints are inequalities and constraints $(m+1)$ to $n$ are equalities, our problem becomes:

Minimize $\phi(x, r)=f(x)+g\left(r_{k}\right) B\left(x_{k}\right)+\frac{1}{g\left(r_{k}\right)} \alpha\left(x_{r}\right)$

The function $\phi(x, r)$ is then minimized for a sequence of monotonically decreasing $\mathrm{r}>0$. (Greig, 1980)

\section{A Mixed Exterior-Interior Point Method:}

We can solve the constrained problem given in eq.(1) to eq.(3) by constructing a new objective function $\phi\left(x, r_{k}\right)$ which is defined in eq. (5).Now our aim is to minimize the function $\phi\left(x, r_{k}\right)$ by starting from a 
feasible point $x_{0}$ and with initial value $r_{0}=1$ and the method reducing $r_{k}$ is simple iterative method such that:

$$
r_{k+1}=\frac{r_{k}}{\mu},
$$

where $\mu$ is a constant equal to 10 and the search direction $d_{k}$ in this case can be defined

$$
d_{k}=-H_{k} g_{k},
$$

where $H_{k}$ is a positive definite symmetric approximation matrix to the inverse Hessian matrix $G^{-1}$ and $g$ is the gradient vector of the function $\phi\left(x, r_{k}\right)$.

The next iteration is set to further point

$$
x_{k+1}=x_{k}+\lambda_{k} d_{k} \text {, }
$$

where $\lambda$ is a scalar chosen in such that $f_{k+1}<f_{k}$. We thus test $c_{i}\left(x_{k+1}\right)$ to see that it is positive for all $i$. We find a feasible $x_{k+1}$ and we can then proceed with the interpolation. Then the matrix $H_{k}$ is updated by a correction matrix to get

$$
H_{k+1}=H_{k}+\phi_{k}
$$

where $\phi_{k}$ is a correction matrix which satisfies quasi-Newton condition namely $\left(H_{k+1} y_{k}=\rho v_{k}\right)$ where $v_{k}$ and $y_{k}$ are difference vector between two successive points and gradients, respectively and $\rho$ is any scalar.

The initial matrix $H_{0}$ chosen to be identity matrix $I . H_{k}$ is updated through the formula of BFGS update. (Fletcher, 1970).

$$
H_{k+1}=H_{k}^{(1)}+H_{k}^{(2)}
$$

where

$$
\begin{gathered}
H_{k}^{(1)}=H_{k}-\frac{H_{k} y_{k} y_{k}^{T} H_{k}}{y_{k}^{T} H_{k} y_{k}}+w w^{T} \\
H_{k}^{(2)}=\frac{v_{k} v_{k}^{T}}{v_{k}^{T} y_{k}}
\end{gathered}
$$

and

$$
w=\left(y_{k}^{T} H_{k} y_{k}\right)^{0.5}\left(\frac{v_{k}}{v_{k}^{T} y_{k}}-\frac{H_{k} y_{k}}{y_{k}^{T} H_{k} y_{k}}\right)
$$

and terminate of the method if

$$
\left|x_{i}-x_{i-1}\right|<\varepsilon
$$


where $\varepsilon=0.000001$, and

$$
r_{k+1}=\frac{r_{k}}{10}
$$

(Bazarra and Shetty, 2000)

\section{The Interior-Exterior Method: ( Nicholas et al., 1997)}

Step (1): Find an initial approximation $x_{0}$ in the interior of the feasible region for the inequality constraints i.e. $g_{i}\left(x_{0}\right)<0$.

Step (2): Set $k=1$ and $r_{0}=1$ is the initial value of $\mathrm{r}_{\mathrm{k}}$.

Step (3): Set $d_{k}=-H_{k} g_{k}$

Step (5): Set $x_{k+1}=x_{k}+\lambda_{k} d_{k}$, where $\lambda$ is a scalar.

Step (6): Check for convergence i.e. if eq.(14) is satisfied then stop.

Step (7): Otherwise, set $r_{k+1}=\frac{r_{k}}{10}$ and take $x=x^{*}$ and set $k=k+1$ and go to Step 3.

\section{The New Interior-Exterior Method:}

The numerical value of $r_{k}$ has to be chosen carefully in order to achieve a faster convergence. The exterior method $g\left(r_{k}\right) \alpha(x) \rightarrow 0$ as $g\left(r_{k}\right) \rightarrow \infty$, and interior method $g\left(r_{k}\right) B(x) \rightarrow 0$ as $g\left(r_{k}\right) \rightarrow 0$ in order to make a balance for two methods, we have to find $r_{k}$ such that depend on $\alpha(x), B(x)$

The initial value $r_{0}$ which is derived as

$$
\begin{aligned}
& \phi\left(x, r_{k}\right)=f(x)+\sinh \left(r_{k}\right) B(x)+\frac{1}{\sinh \left(r_{k}\right)} \alpha(x) \\
& =f(x)+\sinh \left(r_{k}\right) \frac{1}{c_{k}}+\frac{1}{\sinh \left(r_{k}\right)}\left[h_{k}\right]^{2} \\
& \nabla \phi\left(x, r_{k}\right)=\nabla f(x)-\sinh \left(r_{k}\right) \frac{\nabla c_{k}}{\left[c_{k}\right]^{2}}+\frac{2 h_{k} \nabla h_{k}}{\sinh \left(r_{k}\right)}
\end{aligned}
$$

Such that $\nabla \phi\left(x, r_{k}\right)=0$

We have

$$
=\nabla f(x)-\sinh \left(r_{k}\right) \frac{\nabla c_{k}}{\left[c_{k}\right]^{2}}+\frac{2 h_{k} \nabla h_{k}}{\sinh \left(r_{k}\right)}=0
$$

If we take $\sinh \left(r_{k}\right)>0$, then we have

$$
\sinh \left(r_{k}\right) \nabla f(x)-\sinh ^{2}\left(r_{k}\right) \frac{\nabla c_{k}}{\left[c_{k}\right]^{2}}+2 h_{k} \nabla h_{k}=0
$$


This implies

$$
\begin{gathered}
\sinh ^{2}\left(r_{k}\right) \frac{\nabla c_{k}}{\left[c_{k}\right]^{2}}-\sinh \left(r_{k}\right) \nabla f(x)-2 h_{k} \nabla h_{k}=0 \\
\sinh \left(r_{k}\right)=\frac{\nabla f(x) \mp \sqrt{[\nabla f(x)]^{2}+8 h_{k} \nabla h_{k} \frac{\nabla c_{k}}{\left[c_{k}\right]^{2}}}}{2 \frac{\nabla c_{k}}{c_{k}^{2}}}
\end{gathered}
$$

If three points can be selected to find the minimum value of $r_{k}$, it is usually sufficient to approximate the function with the quadratic eq.(22), the optimum value of $r$ is then given by one of the following roots to eq.(22).

$$
r_{\min }=\sinh ^{-1}\left[\frac{\nabla f(x) \mp \sqrt{[\nabla f(x)]^{2}+8 h_{k} \nabla h_{k} \frac{\nabla c_{k}}{\left[c_{k}\right]^{2}}}}{2 \frac{\nabla c_{k}}{c_{k}^{2}}}\right]
$$

$$
\text { Let } A=\frac{\nabla f(x) \mp \sqrt{[\nabla f(x)]^{2}+8 h_{k} \nabla h_{k} \frac{\nabla c_{k}}{\left[c_{k}\right]^{2}}}}{2 \frac{\nabla c_{k}}{c_{k}^{2}}}
$$

Then

$$
r_{\text {min }}=\sinh ^{-1}(A)=\ln \left(A+\sqrt{A^{2}+1}\right)
$$

In the above suggestion corresponding to the assumption for deriving a new parameter to make a balance between the previous method, we have suggested the following new method.

\section{The New Proposed Method:}

Step (1): Find an initial approximation $x_{0}$ in the interior of the feasible region for the inequality constraints i.e. $g_{i}\left(x_{0}\right)<0$.

Step (2): Set $k=1$.

Step (3): Find the initial value of $r_{o}$ by using eq.(25).

Step (4): Set $d_{k}=-H_{k} g_{k}$

Step (5): Set $x_{k+1}=x_{k}+\lambda_{k} d_{k}$, where $\lambda$ is a scalar.

Step (6): Check for convergence i.e. if eq.(14) is satisfied then stop.

Otherwise go to step 7. 
Step (7): Set $r_{k+1}=\frac{r_{k}}{10}$ and take $x=x *$ and set $k=k+1$ and go to step 4 .

\section{Results and Calculations:}

In order to assess the performance of the new method is tested over (6) non-linear test functions with $1 \leq n \leq 3$ and $1 \leq c_{i}(x) \leq 7$.

All the results are obtained using pentium 3. All programs are written in FORTRAN language and for all cases the stopping criterion taken to be

$$
\left|x_{i}-x_{i-1}\right|<\delta, \quad \delta=10^{-5}
$$

In this paper, the two methods used the same exact line search strategy which is the quadratic interpolation technique directly adapted from (Bunday, 1984).

The comparative performance for the two methods is evaluated by considering NOF, NOI, and NOG, where NOF is the number of function evaluations, NOI is the number of iterations and NOG is the number of gradient evaluations.

\section{Discussion:}

In table (1), we have compared our new method with exteriorinterior point method.

From table (2), it is clear that taking exterior-interior point algorithm, as the standard (100\%). The new method has an improvement on the standard exterior-interior point method in about (18\%) NOF, $(20 \%) \mathrm{NOI}$ and $(17 \%)$ NOG.

Table (1)

Comparative performance of the two algorithms

\begin{tabular}{|c|c|c|c|c|c|c|}
\hline \multirow[t]{2}{*}{ Test function } & \multicolumn{3}{|c|}{ Exterior-interior algorithm } & \multicolumn{3}{|c|}{ New algorithm } \\
\hline & NOI & (NOF) & NOG & NO & $(\mathrm{NOF})$ & NOG \\
\hline 1. & 2 & (164) & 56 & 2 & $(50)$ & 30 \\
\hline 2. & 10 & (795) & 127 & 8 & (715) & 105 \\
\hline 3. & 10 & $(807)$ & 131 & 8 & (798) & 114 \\
\hline 4. & 5 & (2726) & 304 & 3 & (1929) & 203 \\
\hline 5. & 7 & (99) & 34 & 4 & (96) & 34 \\
\hline 6. & 10 & $(\mathbf{8 0 3})$ & 138 & 10 & (901) & 146 \\
\hline Total & 44 & (5394) & 790 & 35 & (4489) & 632 \\
\hline
\end{tabular}

Table (2)

Improvement Ratio New

\begin{tabular}{|c|c|c|}
\hline & Exterior-interior algorithm & New algorithm \\
\hline NOF & $100 \%$ & $\mathbf{8 3 . 3}$ \\
\hline NOI & $100 \%$ & $\mathbf{7 9 . 5}$ \\
\hline
\end{tabular}


7. Appendix:

Test functions:

1. $\min f(x)=x_{1}^{2} x_{2}$

s.t.

$$
\begin{aligned}
& x_{1} x_{2}-\left(\frac{x_{1}^{2}}{2}\right)=6 \\
& x_{1}+x_{2} \geq 0
\end{aligned}
$$

Bazaraa, (2000)

2. $\min f(x)=\left(x_{1}-2\right)^{2}+\left(x_{2}-1\right)^{2}$

s.t.

$$
\begin{gathered}
x_{1}-2 x_{2}=-1 \\
\frac{-x_{1}^{2}}{4}+x_{2}^{2}+1 \geq 0
\end{gathered}
$$

3. $\min f(x)=x_{1} x_{2}$

s.t.

$$
\begin{gathered}
25-x_{1}^{2}-x_{2}^{2}=0 \\
x_{1}+x_{2} \geq 0
\end{gathered}
$$

4. $\min f(x)=x_{1} x_{4}\left(x_{1}+x_{2}+x_{3}\right)+x_{3}$

s.t.

$$
\begin{aligned}
& x_{1}^{2}+x_{2}^{2}+x_{3}^{2}+x_{4}^{2}=40 \\
& x_{1} x_{2} x_{3} \geq 25 \\
& 5 \geq x_{i} \geq 1
\end{aligned}
$$

5. $\min f(x)=x_{1}^{2}+x_{2}^{2}$

s.t.

$$
\begin{aligned}
& x_{1}+2 x_{2}=4 \\
& x_{1}^{2}+x_{2}^{2} \leq 5 \\
& x_{i} \geq 0
\end{aligned}
$$

6. $\min f(x)=\left(x_{1}-3\right)^{2}+\left(x_{2}-2\right)^{2}$

s.t.

$$
\begin{aligned}
& x_{1}+2 x_{2}=4 \\
& x_{1}^{2}+x_{2}^{2} \leq 5 \\
& x_{i} \geq 0
\end{aligned}
$$

( see Gottefered, 1973) 


\section{REFERENCES}

[1] Adby, P.R. and Dempster, A. (1983) “Introuduction to Optimization Methods", London, Oxford, Chapman and Hall.

[2] Bazaraa, S. and Shetty, C.M. (2000) "Nonlinear Programming: Theory and Algorithms" Newyork, Chichester, Brisbance, Torant.

[3] Biggs, M.C. (1989) "Halfield Polytechnic Numerical Optimization", The Optima Package, Special Communication.

[4] Bunday, B.D. (1984) "A Basic Optimization Methods", Edward Arnold, Bedford Square, London.

[5] Fletcher, R., (1970) "A New Approach to Variable Metric Algorithms", the Computer Journal, 13, PP.317-322.

[6] Gottefered, B.S. and Weisman, J., (1973) "Introduction to Optimization Theory", Prentice-Hall, Englewood Cliffs, N.J.

[7] Greig, M.D. (1980) "Optimization", Longman, London and Newyork.

[8] Nicholas, J.M. and Gould and Philippe, L. Toint, (1997) "A Note on the Second Order Convergence of Optimization Algorithms Using Barrier Function". Watson Research Center, USA.

[9] Toint, Ph. L.; Cann, A.R.; Gould, N., (1994) "Large Scale Nonlinear Constrained Optimization": A current survey, Report 94/1, IBM T.J. Watson Research Center, USA. 